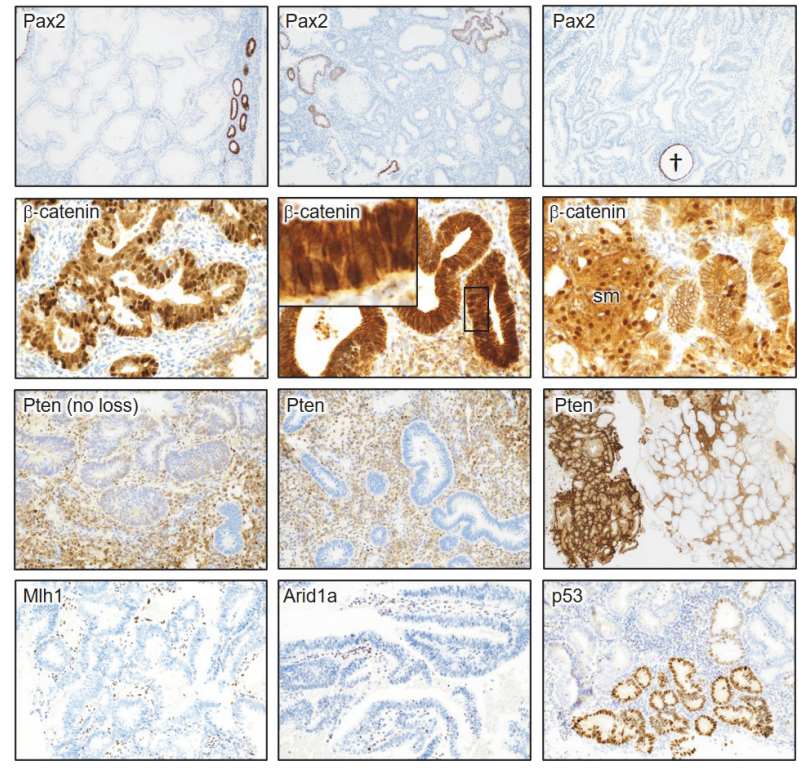

Abstract 496 Figure 1

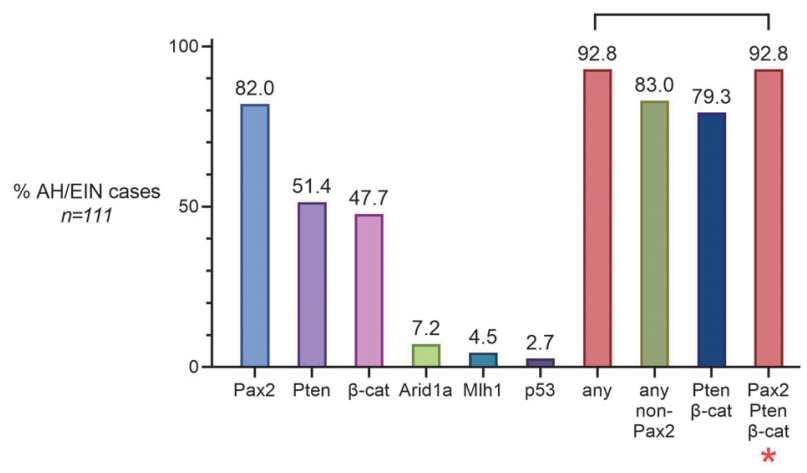

Abstract 496 Figure 2

patients were analyzed by immunohistochemistry for 6 markers: Pax2, Pten, Mlh1, b-catenin, Arid1a, and p53. Aberrant expression consistent with an underlying molecular defect was tabulated for each case and marker. An additional set of $\mathrm{n}=79$ normal endometria was also analyzed to define optimal criteria for marker aberrance. The performance characteristics of each marker, the entire panel, and subsets thereof were statistically analyzed.

Result(s)* In order of number of cases detected, the most frequently aberrant markers in AH/EIN were Pax2 $(82.0 \%$ of cases), Pten (51.4\%), b-catenin (47.7\%), Arid1a (7.2\%), Mlh1 $(4.5 \%)$, and p53 (2.7\%). The great majority of cases showed aberrant expression of $\geq 2$ markers. The 6 markers together identified $92.8 \%$ of cases. Arid1a and Mlh1 proved to be robust and readily-scored markers, but all cases showing aberrant expression of either of these two markers was also detected by b-catenin, Pax2, or Pten.

Conclusion* A limited panel of only 3 markers (Pax2, Pten, and b-catenin) showed optimal performance characteristics as a diagnostic adjunct in the histopathologic diagnosis of AH/EIN. Use of this panel was practicable and robust, with at least one of the 3 markers being aberrant in $92.8 \%$ of $\mathrm{AH} / \mathrm{EIN}$.

\section{A CASE REPORT OF BENIGN METASTASIZING LEIOMYOMA}

${ }^{1} G$ Vinoya*, 2J Tan. 'Tarlac provincial hospital, ob-gyne, Tarlac City, Philippines; ${ }^{2}$ Tarlac provincial hospital, Obstertrics and gynaecology, Tarlac City, Philippines

\subsection{6/ijgc-2021-ESGO.527}

Introduction/Background* Benign metastasizing leiomyoma $(\mathrm{BML})$ is a rare benign condition involving the extra-uterine spread of smooth muscle cells with histological, immunological, and molecular patterns similar to those of benign uterine leiomyomas. Due to the uncommon nature of the disease, diagnosis and treatment is very challenging. There are no current standardized guidelines for treatment of BML but usually treated with a combination of surgical resection and radiotherapy, followed by hormonal treatment and monitored through radiography.

Methodology This is a case of a 49 year old G0 with an incidental finding of BML in the lungs. Routine pre-employment chest $\mathrm{x}$-ray revealed pulmonary nodules. Computed tomography (CT) scan of the abdomen revealed uterine leiomyoma. Video-assisted thoracic surgery (VATS) with wide resection of pulmonary nodules and frozen section was done. Histopathology revealed BML. Immunostains for p53, Ki-67 and p16 were negative, while immunostain for estrogen and progesterone receptor (ER/PR) were positive. Serial chest radiography monitoring showed no significant interval change in pulmonary nodules and patient remained asymptomatic. Few years later, the patient presented an enlarged abdomen. Transvaginal ultrasound revealed a pedunculated subserous myoma. The patient underwent exploratory laparotomy, extrafascial hysterectomy with bilateral salpingooophorectomy. Histopathology revealed BML.

Result(s)* Currently, patient has been asymptomatic for 6 years with no interval changes in pulmonary nodules size despite having multiple lesions in both lungs.

Conclusion* BML is a monoclonal benign tumor with high metastatic potential. A hormone dependent, which growth depends primarily on estrogen and progesterone. This can metastasize to distant organs commonly in the lung (PBML), hence, confused with leiomyosarcoma. Uterine surgeries such as curettage, hysteromyomectomy and hysterectomy can cause it to spread hematogenously to the lung through venous circulation. Patients are usually asymptomatic at presentation and lesions are only incidentally discovered during routine physical examinations as multiple pulmonary nodules in chest radiography. Thoracoscopic lung biopsy remains the gold standard in the diagnosis for PBML. The most helpful pathologic features that characterize PBML and other BMLs are their low cellularity and mitotic index, and the absence of nuclear atypia and tumor necrosis. Immunohistochemistry staining usually is positive for Desmin, Smooth Muscle Actin (SMA) and ER/PR. 


\section{A Case Report of Benign Metastasizing Leiomyoma (BML) Gretel Beau L. Vinoya, MD, Jaynet D. Tan, MD, FPOGS, FSGOP \\ Tarlac Provincial Hospital}

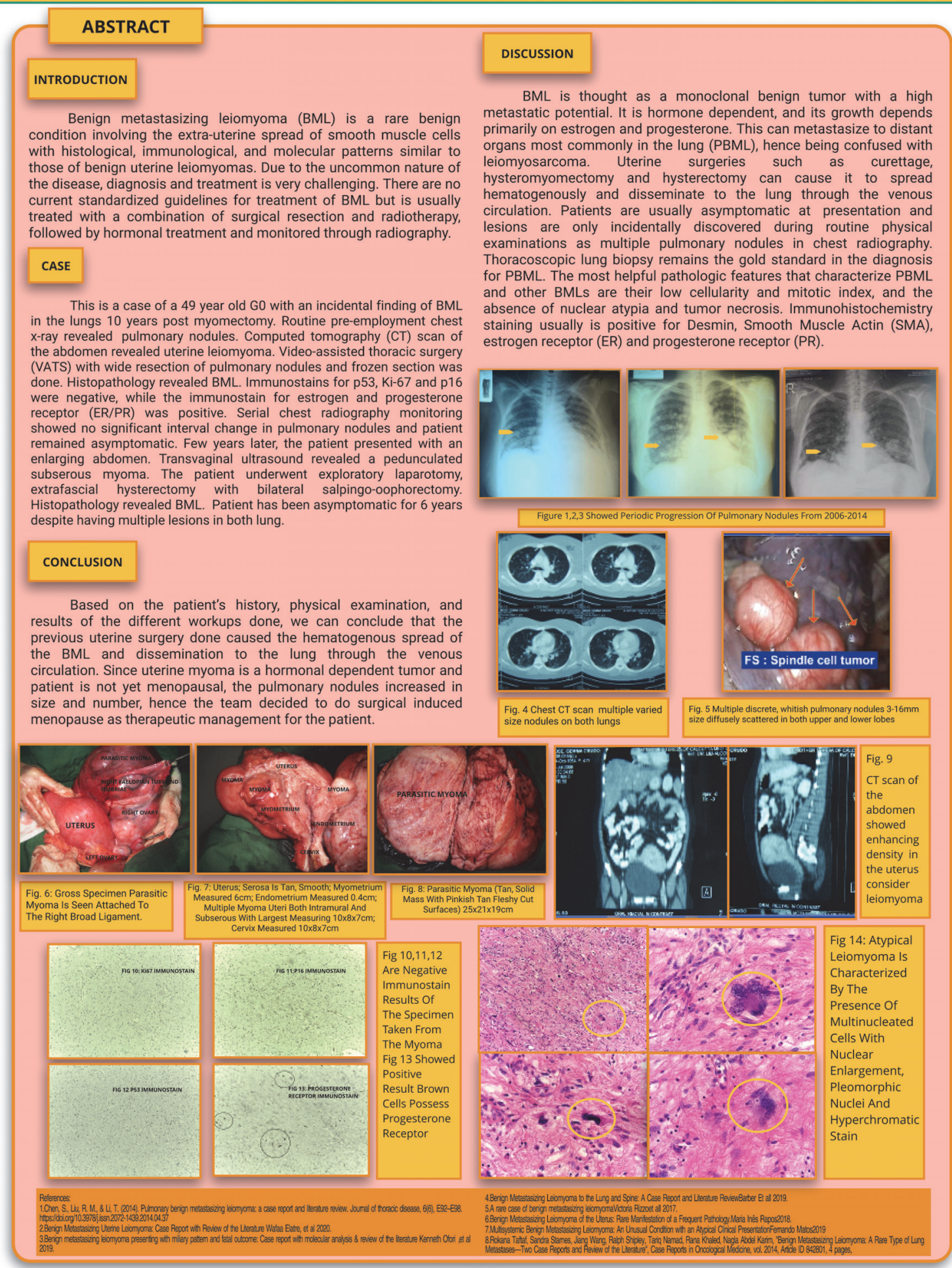

Abstract 672 Figure 1 